\title{
O uso da Curcumina (Curcuma Longa) no tratamento periodontal
}

\author{
The use of Curcumin (Curcuma Longa) in periodontal treatment
}

\author{
Ana LuZia Araújo Batista ${ }^{1}$ \\ Maria Helena Chaves de Vasconcelos Catão
}

\begin{abstract}
RESUMO
Introdução: A doença periodontal (DP) é um importante problema de Saúde Pública sem tratamento eficiente, apesar das diversas modalidades disponíveis. A fototerapia e a fitoterapia representam opções terapêuticas. Dentre os fitoterápicos usados em Periodontia, a Curcuma Longa apresenta propriedades anti-inflamatórias, antibacterianas, antioxidantes e adstringentes, com melhora significativa na saúde gengival. Dessa forma, é uma alternativa antibacteriana potencial e promissora por seus efeitos diretos ou por atuar como fotossensibilizador (FS) na Terapia Fotodinâmica (TFD). Objetivo: Portanto, o objetivo desta revisão literária foi buscar artigos científicos que mencionassem essa temática. Material e Métodos: Foi realizada uma busca no banco de dados da PUBMED, tendo como critério inicial "texto completo" e "últimos 10 anos", utilizando as seguintes palavras-chave: "curcumin and periodontitis" e "curcumin and periodontal treatment". Resultados: Da busca realizada, foram totalizados 22 artigos e 17 artigos, respectivamente. Conclusão: Pode-se concluir que a curcumina representa um auxiliar no tratamento periodontal, juntamente à raspagem e alisamento radicular (RAR), com benefício equivalente à clorexidina e com efeitos colaterais mínimos. As mudanças na formulação e nas formas de apresentação são propostas de melhorar a biodisponibilidade e, por conseguinte, a eficácia da curcumina.
\end{abstract}

Palavras-chave: Curcumina. Periodontite. Terapia combinada.

\begin{abstract}
Introduction: Periodontal disease (PD) is an important public health problem without effective treatment, despite the different modalities available. Phototherapy and herbal medicine represent therapeutic options. Among the phytotherapics used in Periodontics, Curcuma Longa has anti-inflammatory, anti-bacterial, antioxidant and astringent properties, with significant improvement in gingival health. In this way, it is a potential and promising antibacterial alternative for its direct effects or for acting as a photosensitizer (FS) in Photodynamic Therapy (PDT). Objective: Therefore, the objective of this literary review was to search for scientific articles that mention this theme. Material and Methods: A search was performed on the PUBMED database, using the following keywords: "full text" and "last 10 years" using the following keywords: "curcumin and periodontitis" and "curcumin and periodontal treatment". Results: Of the search carried out, 22 articles and 17 articles were totaled, respectively. Conclusion: It can be concluded that curcumin is an adjunct to periodontal treatment, together with scaling and root planning (RAR), with a benefit equivalent to chlorhexidine and with minimal side effects. Changes in formulation and presentation are proposed to improve the bioavailability and consequently the efficacy of curcumin.
\end{abstract}

Keywords: Curcumin. Periodontitis. Combined modality therapy.

Doutoranda no Programa de Pós-Graduação em Odontologia da Universidade Estadual da Paraíba, Campina Grande, Paraíba, Brasil Professora Doutora no Programa de Pós-Graduação em Odontologia da Universidade Estadual da Paraíba, Campina Grande, Paraíba, Brasil 


\section{INTRODUÇÃO}

A doença periodontal (DP) é um importante problema de Saúde Pública, com grande impacto socioeconômico. ${ }^{1}$ A gengivite é a forma mais prevalente, afetando mais de $80 \%$ da população mundial, e tem seu início com o biofilme patogênico ao redor dos dentes e a produção de metabólitos destrutivos, podendo progredir para um quadro de periodontite, em indivíduos suscetíveis. ${ }^{2,3}$

A periodontite tem uma prevalência de $5 \%$ a $30 \%$ na população adulta, sendo caracterizada por inflamação progressiva do tecido gengival, com alterações patológicas nos tecidos de sustentação dentária, levando à perda irreversível de estruturas periodontais e à perda dentária gradual em estágios posteriores. . $^{4-9}$

A terapia periodontal tem como objetivo promover a cura do tecido inflamado, eliminar as bolsas doentes, reduzir o número de bactérias patogênicas, remover o biofilme e todos os fatores que favorecem seu acúmulo e alterar as condições ambientais dos nichos microbianos. ${ }^{10-12}$ Nenhum protocolo de tratamento é conhecido por ser capaz de controlar totalmente a doença periodontal, ${ }^{3}$ ou seja, ainda não existe um tratamento eficiente. ${ }^{1}$

A maioria das modalidades de tratamento periodontal tenta deter o avanço da destruição periodontal. Mas, mesmo com a aplicação de modalidades periodontais apropriadas, há algumas condições ou situações que permanecem inalteradas. ${ }^{7} \mathrm{~A}$ fototerapia e a fitoterapia são opções terapêuticas no tratamento de periodontite..$^{13}$

A Terapia Fotodinâmica (TFD) utiliza diferentes fontes de luz e de fotossensibilizador (FS), representando uma alternativa antibacteriana potencial por significar uma nova ferramenta para combater bactérias patogênicas. ${ }^{14}$ A curcumina, além de seus efeitos diretos, pode atuar como um FS na TFD e apresenta atributos ideais como a capacidade de formar oxigênio singleto e excelente biocompatibilidade, ${ }^{3,14-16}$ além de suas propriedades anti-inflamatórias, antibacterianas, antioxidantes e adstringentes, com melhora significativa na saúde gengival. ${ }^{17}$

Portanto, o objetivo dessa revisão de literatura foi buscar artigos no banco de dados da PUBMED, no período de janeiro e fevereiro de 2019, tendo como critério inicial "texto completo" e "últimos 10 anos", utilizando as palavras-chave: "curcumin and periodontitis" e "curcumin and periodontal treatment" para avaliar os tipos de estudo e as conclusões expostas.

\section{Metodologia}

Foi realizada uma busca na literatura no banco de dados da PUBMED, no período de janeiro e fevereiro de 2019 , tendo como critério inicial "texto completo" e "últimos 10 anos". Foram utilizadas as seguintes palavras-chave: "curcumin and periodontitis" e "curcumin and periodontal treatment". Foi realizada a leitura de títulos e resumos para exclusão dos artigos com temática divergente e o confronto dos artigos na busca de repetição. Por fim, os artigos selecionados foram lidos na íntegra.

\section{REVISÃo de LITERATURA}

\section{Doença periodontal}

A DP é uma condição infecciosa e inflamatória crônica comum, de etiologia multifatorial, sendo as bactérias patogênicas o agente etiológico primário. ${ }^{(12,18,19}$ Seu início e a sua progressão estão significativamente associados à liberação de toxinas bacterianas e à resposta inflamatória do hospedeiro, ${ }^{19}$, 20 ou seja, ao desequilíbrio entre a virulência bacteriana e a capacidade de defesa do hospedeiro. ${ }^{21}$ 


\section{PUBMED}

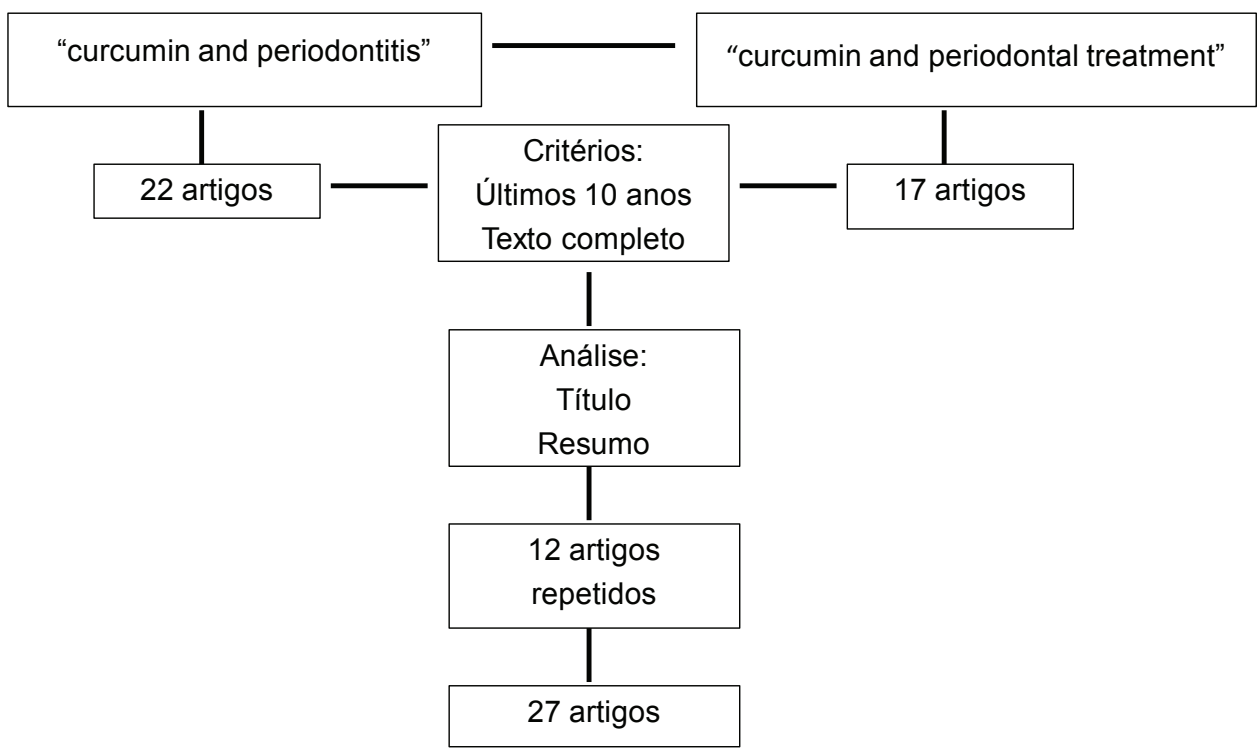

Figura 1: Fluxograma para seleção dos artigos

A infecção periodontal é uma infecção polimicrobiana, em que centenas de diferentes espécies de microrganismos estão presentes. A microbiota na fase de biofilme é até 1000 vezes mais tolerante e/ou resistente a agentes antimicrobianos em comparação com a fase planctônica, além de apresentar 10 a 600 vezes maior taxa de eficiência de transformação, o que pode aumentar a sobrevivência da microbiota. ${ }^{16}$

O biofilme bacteriano patogênico é um dos mais diversos ecossistemas microbianos do ser humano, envolvendo cerca de 700 espécies bacterianas diferentes, principalmente bactérias anaeróbias gram-negativas, sendo as mais proeminentes Porphyromonas gingivalis, Prevotella intermedia, Aggregatibacter actinomycetemcomitans $\mathbf{s}^{1,18,21}$ e Fusobacterium nucleatum. ${ }^{13,14} \mathrm{~A}$ natureza do biofilme da placa dentária fornece um ambiente especializado para os microrganismos, garantindo assim a sua vitalidade e patogenicidade..$^{10}$

A agregação bacteriana raramente leva a uma infecção evidente, mas pode envolver uma resposta imuneinflamatória, que é responsável pela perda de inserção clínica e destruição do tecido conjuntivo, osso alveolar, cemento e ligamento periodontal, com aumento da profundidade da bolsa, levando, eventualmente, à perda de dentes. ${ }^{3,8,18}$

Embora as bactérias sejam essenciais para a doença periodontal, a maioria dos danos nos tecidos é causada por mediadores inflamatórios e radicais livres, ${ }^{2}$ sendo que a resposta do sistema imunológico do hospedeiro frente a bactérias patogênicas é responsável pelo início de uma inflamação imunomediada. ${ }^{1}$ As espécies reativas de oxigênio (EROs) são produzidas a partir dos tecidos periodontais inflamados por ativação funcional dos neutrófilos polimorfonucleares (PMNs), ${ }^{7} \mathrm{com}$ efeitos deletérios nas células do tecido quando produzidas em excesso. ${ }^{12}$

A resposta imune do hospedeiro identifica patógenos invasores e tenta neutralizar ou eliminar os microrganismos. Uma vez iniciada a resposta do hospedeiro, vários mediadores pró-inflamatórios, como interleucinas (IL) 
e fator de necrose tumoral alfa (TNF- $\alpha$ ), citocinas, prostaglandinas e metaloproteinases da matriz (MPM) são liberados de leucócitos, fibroblastos ou outras células. Dessa forma, uma resposta imune inadequada leva à superprodução desses mediadores e, por conseguinte, dano ao tecido conjuntivo, com perda de inserção periodontal e destruição óssea por reabsorção. 2,6,13,20

\section{Tratamento Periodontal}

Como a principal causa de doença periodontal é o biofilme patogênico, devese priorizar o meio mais efetivo e econômico de controle em bolsas periodontais rasas, na tentativa de impedir a progressão da doença. ${ }^{4}$ O meio mais eficiente deve ser não invasivo, com a utilização de medidas preventivas para evitar o estabelecimento da doença e seus efeitos deletérios e impedir a formação e desenvolvimento do biofilme. ${ }^{1}$

As modalidades de tratamento para a resolução da inflamação periodontal podem ser cirúrgicas, não cirúrgicas ou a combinação de ambas, com base na severidade da destruição periodontal. A terapia não cirúrgica inclui métodos mecânicos e quimioterápicos para minimizar ou eliminar o biofilme microbiano, ${ }^{8}$ além do uso de antibióticos. ${ }^{11}$

A modalidade terapêutica de rotina é a raspagem e alisamento radicular (RAR), que consiste na remoção de biofilme e cálculo supra e subgengival, retornando assim os tecidos a um estado de saúde. 5,9-11,21 No entanto, a recolonização dos locais tratados pode ocorrer ${ }^{10}$ ou até mesmo pode não conseguir eliminar completamente as bactérias patogênicas, ${ }^{3,8}$ sendo os agentes farmacológicos usados como adjuvantes da terapia mecânica, ${ }^{10}$ seja com entrega local ou de administração sistêmica. ${ }^{(19)}$ As bolsas profundas requerem um regime antimicrobiano adjuvante para auxiliar no desbridamento mecânico das superfícies dos dentes pela liberação local e sistêmica de agentes químicos. ${ }^{17}$
Após a terapia não cirúrgica, várias bolsas periodontais profundas podem persistir e, nesses casos, o tratamento consiste em procedimentos cirúrgicos. ${ }^{5}$ Embora a maioria dos microrganismos patogênicos após a cirurgia possa sobreviver, o uso subsequente de agentes antimicrobianos pode melhorar os resultados. ${ }^{1}$

Ogluconatodeclorexidina(CHX)éumagente bactericida eficaz e um fármaco antimicrobiano de amplo espectro e considerado o padrãoouro na manutenção da saúde periodontal por suas características de segurança, eficácia, substantividade e falta de toxicidade, além de não produzir microrganismos resistentes e destruir outras categorias de microrganismos com pouco risco de desenvolvimento de infecções oportunistas. ${ }^{19}$ No entanto, após o uso prolongado, pode levar a efeitos colaterais indesejáveis, como a pigmentação dos dentes e dos tecidos orais. ${ }^{5}$

A antibioticoterapia é uma medida amplamente utilizada, mas seu efeito é limitado a curto prazo e, devido à necessidade de altas doses, pode promover o desenvolvimento de resistência bacteriana, efeitos colaterais, interações medicamentosas, reações alérgicas, problemas gastrointestinais e inconsistência na adesão do paciente. .,3,8,21 $^{-1}$

O aparecimento de resistência aos antibióticos e o risco de reações adversas secundárias para o uso de antibióticos têm desenvolvido uma necessidade para a introdução de um método de substituição para remoção de microrganismos patogênicos. ${ }^{(13)}$ Para evitar essas limitações de administração sistêmica, foi introduzida uma abordagem diferente que usa sistemas de distribuição local que contêm agentes antibióticos ou antissépticos. ${ }^{21}$

A administração local de antimicrobianos parece um meio eficaz devido à natureza sítio-específica das infecções periodontais e pelas vantagens que incluem o envolvimento 
sistêmico mínimo, menos efeitos colaterais sistêmicos, menor resistência a drogas, maior penetração do medicamento no local, melhor adesão do paciente e o desconforto mínimo, com liberação local por um período prolongado em um nível farmacológico estável ${ }^{8,10,17}$ e por atingir uma concentração 100 vezes maior em comparação com um regime sistêmico de drogas. ${ }^{19}$

Vários agentes antibacterianos, como clorexidina $(\mathrm{CHX})$, metronidazol, tetraciclinas, iodo-povidona, o ornidazol, a doxiciclina, a minociclina, produtos fitoterápicos, fluoreto estanhoso e outros têm sido aplicados subgengivalmente. . $, 8,10,12$

A fototerapia é um dos substitutos úteis para a terapia de antibióticos no tratamento de periodontite ${ }^{13}$ e a Terapia Fotodinâmica (TFD) é uma alternativa antibacteriana potencial por representar uma nova ferramenta para combater bactérias multirresistentes e selvagens, ${ }^{14}$ com uma técnica não invasiva ${ }^{16}$ que auxilia em locais de difícil acesso. ${ }^{15}$ Esse modelo terapêutico envolve a utilização de um fotossensibilizador (FS) que é ativado por exposição à luz de um comprimento de onda específico, na presença de oxigênio. 3,13,15

As abordagens preventivas e de tratamento atuais são apenas parcialmente eficazes, pois se concentram principalmente no controle do biofilme, ao invés de controlar os mediadores inflamatórios do hospedeiro responsáveis pelo dano tecidual. ${ }^{(2)}$ A terapia modulatória do hospedeiro é uma estratégia terapêutica plausível para a doença periodontal, principalmente dirigidos à inibição de citocinas específicas por atenuar a progressão da doença periodontal e aumentar as respostas terapêuticas, ${ }^{18}$ uma vez que a resposta do hospedeiro que é essencialmente protetora por intenção, paradoxalmente também pode resultar em dano tecidual. ${ }^{6}$

As doenças periodontais também podem ser tratadas com terapia antioxidante como parte do protocolo por eliminar os radicais livres e prevenir os danos causados por eles, agindo, portanto, como agentes corretivos e profiláticos. O aconselhamento nutricional e a suplementação devem ser uma parte importante do protocolo periodontal para melhorar o resultado da terapia periodontal convencional. ${ }^{7}$

A doença periodontal aponta para a necessidade de manejo efetivo e eficiente, na tentativa de restringir seu impacto na saúde geral e na qualidade de vida do paciente, ${ }^{4}$ com uma abordagem preventiva e de tratamento que inclua uma abordagem combinada em relação à modulação do hospedeiro, à resolução da inflamação e ao manejo direto de bactérias. ${ }^{2}$

\section{Curcumina}

A Medicina Ocidental obteve sucesso na prevenção e no tratamento de uma variedade de doenças orais. Por esse motivo, apesar do progresso no desenvolvimento da Ciência Médica, as plantas continuam a ser uma importante fonte de drogas. Assim, a busca por produtos alternativos continua, e os fitoquímicos naturais isolados de plantas são considerados boas alternativas aos produtos químicos sintéticos. ${ }^{8,20}$

Atualmente, o uso de produtos fitoterápicos em Odontologia é cada vez maior. Isso pode ser atribuído à sua fácil disponibilidade, baixo custo e menores efeitos colaterais. ${ }^{(10)}$ Plantas medicinais têm sido usadas como um agente de tratamento tradicional para inúmeras doenças humanas, sendo a cúrcuma (Curcuma longa) um desses produtos naturais que possui valor medicinal. ${ }^{12}$

A Curcuma longa Linn., também conhecida como açafrão-da-índia, é uma erva amplamente utilizada que é distribuída por regiões tropicais e subtropicais do mundo e amplamente cultivada na Índia, China e Indonésia, sendo um membro da família do gengibre, Zingaberaceae. ${ }^{6,7,10,12,21}$ 
Os curcuminoides são responsáveis pela cor amarela da cúrcuma e são divididos em três principais: diferucilmetano (77\%), desmetoxicurcumina (17\%) e bisdemetoxicurcumina $(3 \%),{ }^{4,12}$ bem como, óleos voláteis (turmerona, atlantona $e$ zingiberona), açúcares, proteínas e resinas. ${ }^{21,22}$

A curcumina [1,7-bis-(4-hidroxi3-metoxifenil)-1,6-heptadieno-3,5-diona], (diferucilmetano), é um pigmento fenólico amarelo, extraído da raiz da cúrcuma e o principal componente bioativo., ${ }^{4,719-23} \mathrm{~A}$ curcumina tem sido utilizada na cosmética e como aditivo alimentar, tempero dietético e suplemento fitoterápico por causa de suas potenciais propriedades medicinais. .,9, $19,21,24^{2}$

A curcumina apresenta amplo espectro de efeitos terapêuticos, como propriedades imunoestimulantes, anti-inflamatória, antioxidante, antibacteriana, antiviral, antifúngica, antitumoral, antiespasmódica, hepatoprotetora, antialérgica, antimutagênica, anticoagulante, antifertilidade, antidiabético, curativas, antiprotozoário, antifibrótico, antiveneno, antiulceroso, potenciador da cicatrização, miogênese, hipotensivo, hipocoleste rolêmicas. ${ }^{1,2,5,7,8,13,15,17,19-23}$

A curcumina tem um potencial valor terapêutico, com inúmeras aplicações médicas, principalmente contra várias doenças crônicas, incluindo alergias, artrite reumatoide, diabetes, doenças cardiovasculares, câncer e outras doenças crônicas. ${ }^{18,25}$ Atualmente, a curcumina vem gerando muito interesse no campo da Periodontia, pois pode atingir tanto as bactérias, como sua ação antimicrobiana, assim como, as células inflamatórias do hospedeiro por sua ação anti-inflamatória. ${ }^{2,8}$ Essas propriedades, associadas à ação antioxidante, a tornam uma substância favorável para o tratamento de doenças periodontais. ${ }^{3}$ Embora tenha sido relatada uma série de atividades biológicas e farmacológicas da curcumina, seu potencial terapêutico para doença periodontal destrutiva é pouco compreendido. ${ }^{20}$

\section{Resultado e Discussão}

Da busca realizada, foram totalizados 22 artigos da primeira busca e 17 artigos na segunda. No confronto dos artigos, foram observados que 12 eram repetidos, permanecendo, ao final, 27 artigos. Desse total, dois eram de revisão de literatura e outro de análise estrutural, sendo excluídos da discussão. Dos 24 que se enquadram como pesquisa, quatro deles pode-se considerar como estudo in vivo e in vitro ao mesmo tempo. Com relação aos demais, cinco foram in vitro e 15 in vivo. Desses, 11 foram em humanos e quatro em animais.

A modulação da resposta inflamatória do hospedeiro é uma estratégia terapêutica plausível para a doença periodontal, principalmente dirigidos à inibição de citocinas específicas por atenuar a progressão da doença periodontal e aumentar as respostas terapêuticas. ${ }^{18}$

Numa investigação sobre a resposta inflamatória com a curcumina na DP, Kim et al. $(2011)^{18}$, avaliando a produção de IL-6, Guimarães et al. (2011) ${ }^{20}$, em uma avaliação in vivo, e Guimarães et al. (2012) ${ }^{24}$, na avaliação de outros mediadores como $\left(\mathrm{PGE}_{2}\right), \mathrm{IL}-6 \mathrm{e}$ TNF- $\alpha$ encontraram resultados promissores, com inibição e/ou supressão dos mediadores, sugerindo a curcumina como estratégia terapêutica anti-inflamatória para doenças periodontais.

Mais recentemente, Guru et al., $(2017)^{6}$ avaliaram o efeito anti-inflamatório da curcumina $(1500 \mu \mathrm{g} / \mathrm{ml})$ e seu efeito inibidor sobre a atividade da MMP-9, comparado a doxiciclina $(300 \mu \mathrm{g} / \mathrm{ml})$ e os resultados mostraram que a atividade da MMP-9 foi significativamente diminuída por ambas as drogas e Xiao et al. (2018), ${ }^{9}$ que investigaram os efeitos da curcumina na resposta inflamatória em fibroblastos gengivais, variando a concentração da curcumina 
$(30 \mu \mathrm{g} / \mathrm{g}$ e $100 \mu \mathrm{g} / \mathrm{g})$ e os resultados mostraram atenuação na produção de IL-1 $\beta$ e TNF- $\alpha$.

Os dispositivos de entrega locais usados incluem fibras, verniz, chips, tiras e compactos, géis, micropartículas, filmesenanopartículas. ${ }^{4,11,8}$ Em se tratando de uma nova formulação com curcumina, Gu et al. (2013) ${ }^{25}$ utilizaram uma curcumina quimicamente modificada (CMC2.5), em ratos, com ratos diabéticos com e sem DP e Elburki et al. (2014), ${ }^{26}$ com outro tipo de curcumina modificada (CMC 2.24), analisaram preventivamente os efeitos da administração oral em ratos com periodontite. O primeiro estudo concluiu que o potencial terapêutico da curcumina no tratamento de doenças inflamatórias pode melhorar a inflamação local e também a inflamação sistêmica, enquanto que o segundo estudo apontou uma inibição significativa da perda óssea alveolar, dos mediadores inflamatórios e da destruição de colágeno.

Anitha et al. (2015) $)^{10}$ e Bathia et al. (2014) $)^{17}$ avaliaram a eficácia do gel de curcumina a $1 \%$ com aplicação local no tratamento da periodontite crônica, ambos os estudos como análise dos parâmetros clínicos e microbiológicos em pacientes. No primeiro estudo, o grupo da curcumina apresentou maior redução nos parâmetros clínicos quando comparado ao grupo da clorexidina, enquanto que no segundo, os autores concluíram que a concentração de curcumina utilizada não é sustentada e, portanto, não tem efeitos a longo prazo.

Com o aumento da concentração do gel, como aconteceu na pesquisa de Hosadurga et al. $(2014)^{27}$ com o gel de curcumina $2 \%$, os resultados apresentaram um efeito antiinflamatório por 24-48 horas, o que favorece seu uso como adjuvante útil para melhorar os resultados da terapia periodontal padrão. Anuradha et al. (2015) ${ }^{11}$ e Hugar et al. (2016) ${ }^{5}$ utilizaram em seus estudos o gel de curcumina, mas sem especificar a concentração. No primeiro estudo, puderam inferir que a terapia mecânica, quando combinada com sistemas de entrega local, a exemplo do gel, reduz microrganismos e melhora os parâmetros clínicos quando comparado a RCR isolada e o segundo puderam concluir que o gel de curcumina mostrou ser mais eficaz do que o gel de clorexidina no tratamento de bolsas periodontais leves a moderadas.

Diferentes modos de entrega da curcumina também foram testados. Gottumukkala et al. $(2013)^{4}$ avaliaram a eficácia da irrigação subgengival com curcumina e os resultados mostram uma melhora significativa tanto nos grupos teste e controle, enquanto que Gottumukkala et al. $(2014)^{19}$ avaliaram a eficácia do modo de entrega local de colágeno ancorado em curcumina e a clorexidina mostrou uma melhora maior. Nagasri et al. $(2015)^{21}$ avaliaram o efeito da administração local de curcumina. Houve uma diferença significativa na redução média de perda óssea no local teste e uma redução proporcional das bactérias no grupo teste e controle. Elavarasu et al. (2016) $)^{12}$, por sua vez, avaliaram a eficácia da tira de curcumina $(0,2 \%)$ com redução dos parâmetros clínicos nos locais de teste e controle e atuando como antioxidante no ambiente subgengival.

Em geral, os grupos controle são os que utilizam o padrão-ouro, que é considerado a clorexidina. Mas, Ravishankar et al. (2017) ${ }^{8}$ compararam a eficácia clínica de dois sistemas locais de liberação de fármaco em formas de gel (curcumina e ornidazol) e, nessa avaliação, o grupo de curcumina mostrou uma diminuição significativa nos índices clínicos periodontais.

Em modelos de gengivite experimental, Pulikkotil, Nath (2015) ${ }^{2}$ avaliaram o efeito da curcumina sobre os níveis de IL $\square 1 \beta$ e CCL28 no FCG, e tanto a curcumina quanto a associação da clorexidina-metronidazol foram semelhantes na inflamação clínica e nos níveis de mediadores. Kaur et al. 
$(2017)^{7}$ avaliaram os efeitos da administração sistêmica de curcumina, pimenta e licopeno e os resultados mostram que houve redução dos escores em ambos os grupos, com maior redução no grupo teste.

Sreedhar et al. (2015) ${ }^{15}$ diferenciaram sua pesquisa com a utilização da TFD, usando como FS o gel de curcumina em pacientes. Pôde-se concluir que o uso adjuvante de curcumina em gel como droga subgengival local com RCR tem ação antibacteriana sobre os patógenos periodontais, como $\mathrm{Aa}, \mathrm{Pg}$ e $\mathrm{Pi}$, com efeitos aprimorados com a TFD.

Nessa temática da fototerapia, os estudos in vitro ainda predominam como o de Mahdi et al., $(2015)^{14}$ que compararam os FS (peróxido de hidrogênio, curcumina e eritrosina) e concluíram que qualquer tipo de tratamento pode servir para redução significativa das principais bactérias periopatogênicas e que o efeito bactericida máximo para as espécies examinadas pode ser alcançado pelo tempo de exposição ideal de três minutos. Já Ghanbari et al. $(2015)^{13}$ projetaram um estudo comparando o efeito fototóxico da luz visível e do laser com três FS (eritrosina, curcumina e peróxido de hidrogênio). O resultado do estudo confirma o efeito fototóxico sinérgico da luz visível em combinação com cada um dos FS sobre as bactérias, sendo que a eritrosina e o peróxido de hidrogênio sozinhos tiveram menor efeito tóxico em comparação com a curcumina.

Ainda nesse tipo de estudo, Najafi et al. $(2016)^{3}$ utilizaram curcumina dissolvida em dimetilsulfóxido - DMSO $(0,5 \%)$ com o LED azul e concluíram que a curcumina é uma substância eficaz na prevenção do crescimento de A. actinomycetemcomitans, cujo impacto é reforçado quando usado simultaneamente no procedimento de TFD. Pourhajibagher et al. (2018) ${ }^{16}$ também avaliaram a eficácia da TFD com curcumina na redução do mesmo microrganismo e, com base nos resultados, pode-se afirmar que a curcumina nas concentrações acima de $5 \mu \mathrm{mol} / \mathrm{ml}$ pode reduzir significativamente as UFCs desse periodontopatógeno.

\section{Considerações finaIS}

- A curcumina representa um auxiliar no tratamento periodontal, juntamente à raspagem e alisamento radicular;

- A curcumina apresenta benefício equivalente à clorexidina, representando uma excelente alternativa;

- A curcumina apresenta efeitos colaterais mínimos;

- Sua indicação no tratamento periodontal deve-se principalmente às suas propriedades antissépticas, antimicrobianas, antiinflamatórias e antioxidantes;

- As mudanças na formulação e nas formas de apresentação são propostas de melhorar a biodisponibilidade e, por conseguinte, a eficácia da curcumina.

\section{REFERÊNCIAS}

1. Asteriou E, Gkoutzourelas A, Mavropoulos A, Katsiari C, Sakkas LI, Bogdanos DP. Curcumin for the Management of Periodontitis and Early ACPA-Positive Rheumatoid Arthritis: Killing Two Birds with One Stone. Nutrients. 2018 Jul 16;10(7).

https://www.ncbi.nlm.nih.gov/pubmed/30012973

2. Pulikkotil SJ, Nath $S$. Effects of curcumin on crevicular levels of $\mathrm{IL}-1 \beta$ and CCL28 in experimental gingivitis. Aust Dent J. 2015 Sep;60(3):317-27.

https://onlinelibrary.wiley.com/doi/full/10.1111/ adj.12340

3. Najafi $S$, Khayamzadeh $M$, Paknejad $M$, Poursepanj G, Kharazi Fard MJ, Bahador A. An In Vitro Comparison of Antimicrobial Effects of Curcumin-Based Photodynamic Therapy and Chlorhexidine, on Aggregatibacter actinomycetemcomitans. J Lasers Med Sci. 2016 Winter;7(1):21-5.

https://www.ncbi.nlm.nih.gov/pubmed/27330693 
4. Gottumukkala SN, Koneru S, Mannem S, Mandalapu N. Effectiveness of sub gingival irrigation of an indigenous $1 \%$ curcumin solution on clinical and microbiological parameters in chronic periodontitis patients: A pilot randomized clinical trial. Contemp Clin Dent. 2013 Apr;4(2):186-91.

https://www.ncbi.nlm.nih.gov/pmc/articles/ PMC3757880/

5. Hugar SS, Patil S, Metgud R, Nanjwade B, Hugar SM. Influence of application of chlorhexidine gel and curcumin gel as an adjunct to scaling and root planing: A interventional study. J Nat Sci Biol Med. 2016 Jul-Dec;7(2):149-54.

https://www.ncbi.nlm.nih.gov/pubmed/27433065

6. Guru SR, Kothiwale SV, Saroch N, Guru RC. Comparative evaluation of inhibitory effect of curcumin and doxycycline on matrix metalloproteinase-9 activity in chronic periodontitis. Indian J Dent Res. 2017 SepOct;28(5):560-565.

https://www.ncbi.nlm.nih.gov/pubmed/29072221

7. Kaur S, Sharma R, Sarangal V, Kaur N, Prashar P. Evaluation of anti-inflammatory effects of systemically administered curcumin, lycopene and piperine as an adjunct to scaling and root planing: A clinical study. Ayu. 2017 JulDec;38(3-4):117-121.

https://www.ncbi.nlm.nih.gov/pubmed/30254390

8. Ravishankar PL, Kumar YP, Anila EN, Chakraborty P, Malakar M, Mahalakshmi R. Effect of local application of curcumin and ornidazole gel in chronic periodontitis patients. Int J Pharm Investig. 2017 OctDec;7(4):188-192.

https://www.ncbi.nlm.nih.gov/pubmed/29692978

9. Xiao CJ, Yu XJ, Xie JL, Liu S, Li S. Protective effect and related mechanisms of curcumin in rat experimental periodontitis. Head Face Med. 2018 Aug 16;14(1):12.

https://www.ncbi.nlm.nih.gov/pubmed/30115081

10. Anitha V, Rajesh $P$, Shanmugam M, Priya BM, Prabhu S, Shivakumar V. Comparative evaluation of natural curcumin and synthetic chlorhexidine in the management of chronic periodontitis as a local drug delivery: a clinical and microbiological study. Indian J Dent Res. 2015 Jan-Feb;26(1):53-6.

https://www.ncbi.nlm.nih.gov/pubmed/25961616
11. Anuradha BR, Bai YD, Sailaja S, Sudhakar J, Priyanka M, Deepika V. Evaluation of AntiInflammatory Effects of Curcumin Gel as an Adjunct to Scaling and Root Planing: A Clinical Study. J Int Oral Health. 2015 Jul;7(7):90-3. https://www.ncbi.nlm.nih.gov/pubmed/26229378

12. Elavarasu $S$, Suthanthiran $T$, Thangavelu $A$, Alex S, Palanisamy VK, Kumar TS. Evaluation of superoxide dismutase levels in local drug delivery system containing $0.2 \%$ curcumin strip as an adjunct to scaling and root planing in chronic periodontitis: A clinical and biochemical study. J Pharm Bioallied Sci. 2016 Oct;8(Suppl 1):S48-S52.

https://www.ncbi.nlm.nih.gov/pubmed/27829747

13. Ghanbari H, Mousavi SA, Forouzanfar A, Zakeri M, Shafaee H, Shahnaseri S. Synergic phototoxic effect of visible light or GalliumArsenide laser in the presence of different photo-sensitizers on Porphyromonas gingivalis and Fusobacterium nucleatum. Dent Res J (Isfahan). 2015 Jul-Aug;12(4):323-30.

https://www.ncbi.nlm.nih.gov/pubmed/26288621

14. Mahdi Z, Habiboallh G, Mahbobeh NN, Mina ZJ, Majid Z, Nooshin A. Lethal effect of blue lightactivated hydrogen peroxide, curcumin and erythrosine as potential oral photosensitizers on the viability of Porphyromonas gingivalis and Fusobacterium nucleatum. Laser Ther. 2015 Mar 31;24(2):103-11.

https://www.ncbi.nlm.nih.gov/pubmed/26246690

15. Sreedhar A, Sarkar I, Rajan P, Pai J, Malagi S, Kamath V, Barmappa R. Comparative evaluation of the efficacy of curcumin gel with and without photo activation as an adjunct to scaling and root planing in the treatment of chronic periodontitis: A split mouth clinical and microbiological study. J Nat Sci Biol Med. 2015 Aug;6(Suppl 1):S102-9.

https://www.ncbi.nlm.nih.gov/pubmed/26604595

16. Pourhajibagher $M$, Chiniforush $N$, Monzavi $A$, Barikani H, Monzavi MM, Sobhani S, Shahabi S, Bahador A. Inhibitory Effects of Antimicrobial Photodynamic Therapy with Curcumin on Biofilm-Associated Gene Expression Profile of Aggregatibacter actinomycetemcomitans. J Dent (Tehran). 2018 May;15(3):169-177. https://www.ncbi.nlm.nih.gov/pubmed/30090117 


\section{Ana Luzia Araújo Batista \\ Maria Helena Chaves de Vasconcelos Catão}

17. Bhatia M, Urolagin SS, Pentyala KB, Urolagin SB, K B M, Bhoi S. Novel therapeutic approach for the treatment of periodontitis by curcumin. $\mathbf{J}$ Clin Diagn Res. 2014 Dec;8(12):ZC65-9. https://www.ncbi.nlm.nih.gov/pubmed/25654035

18. Kim SJ. Curcumin suppresses the production of interleukin-6 in Prevotella intermedia $\begin{array}{lll}\text { lipopolysaccharide-activated RAW } 264.7 & \text { RA }\end{array}$ cells. J Periodontal Implant Sci. 2011 Jun;41(3):157-63. https://www.ncbi.nlm.nih. gov/pubmed/21811692

19. Gottumukkala SN, Sudarshan S, Mantena SR. Comparative evaluation of the efficacy of two controlled release devices: Chlorhexidine chips and indigenous curcumin based collagen as local drug delivery systems. Contemp Clin Dent. 2014 Apr;5(2):175-81.

https://www.ncbi.nlm.nih.gov/pubmed/24963242

20. Guimarães MR, Coimbra LS, de Aquino SG, Spolidorio LC, Kirkwood KL, Rossa C Jr. Potent anti-inflammatory effects of systemically administered curcumin modulate periodontal disease in vivo. J Periodontal Res. 2011 Apr;46(2):269-79.

https://www.ncbi.nlm.nih.gov/pubmed/21306385

21. Nagasri $M$, Madhulatha $M$, Musalaiah SV, Kumar PA, Krishna CH, Kumar PM. Efficacy of curcumin as an adjunct to scaling and root planning in chronic periodontitis patients: A clinical and microbiological study. J Pharm Bioallied Sci. 2015 Aug; 7(Suppl 2):S554-8. https://www.ncbi.nlm.nih.gov/pubmed/26538916

22. Nagpal M, Sood S. Role of curcumin in systemic and oral health: An overview. J Nat Sci Biol Med. 2013 Jan;4(1):3-7.

https://www.ncbi.nlm.nih.gov/pubmed/23633828
23. Dhal C, Ahmad FJ, Chauhan A, Jyothi M, Singh RM, Saini PK, Mathur SC, Singh GN. Quality by Design Approach for Simultaneous Estimation of Doxycycline Hyclate and Curcumin by RPHPLC Method. Indian J Pharm Sci. 2015 NovDec;77(6):723-8.

https://www.ncbi.nlm.nih.gov/pubmed/26997700

24. Guimarães MR, de Aquino SG, Coimbra LS, Spolidorio LC, Kirkwood KL, Rossa C Jr. Curcumin modulates the immune response associated with LPS-induced periodontal disease in rats. Innate Immun. 2012 Feb;18(1):155-63.

https://www.ncbi.nlm.nih.gov/pubmed/21242275

25. Gu Y, Lee HM, Napolitano N, Clemens $M$, Zhang Y, Sorsa T, Zhang Y, Johnson F, Golub LM. 4-methoxycarbonyl curcumin: a unique inhibitor of both inflammatory mediators and periodontal inflammation. Mediators Inflamm. 2013;2013:329740.

https://www.ncbi.nlm.nih.gov/pubmed/24453415

26. Elburki MS, Rossa C, Guimaraes MR, Goodenough M, Lee HM, Curylofo FA, Zhang Y, Johnson F, Golub LM. A novel chemically modified curcumin reduces severity of experimental periodontal disease in rats: initial observations. Mediators Inflamm. 2014;2014:959471.

https://www.ncbi.nlm.nih.gov/pubmed/25104884

27. Hosadurga RR, Rao SN, Jose J, Rompicharla NC, Shakil M, Shashidhara R. Evaluation of the efficacy of $2 \%$ curcumin gel in the treatment of experimental periodontitis. Pharmacognosy Res. 2014 Oct;6(4):326-33.

https://www.ncbi.nlm.nih.gov/pubmed/25276071

Submetido em: 13-3-2019

Aceito em: 3-7-2019 